\title{
Influence de la congélation et de la cryodessiccation qui s'ensuit sur le taux de survie et le pourcentage des deux ferments lactiques (culture mixte) : \\ Streptococcus thermophilus - Lactobacillus helveticus
}

par

A. JABARIT

\section{INTRODUCTION}

Dans cette partie de notre étude, nous avons essayé de connaître quelles étaient les influences de la congélation et de la cryodessiccation qui s'ensuit sur le taux de survie et le pourcentage de ces deux ferments lactiques : Streptococcus thermophilus - Lactobacillus helveticus, présentés sous forme de cultures mixtes.

Tandis que le yaourt ordinaire est à base des deux ferments lactiques en symbiose [8 et 9]. Ces derniers sont les suivants : Streptococcus thermophilus - Lactobacillus bulgaricus.

Nous avons donc choisi les ferments helvétiques que nous avons soumis aux différentes températures de congélation variant de $-20^{\circ} \mathrm{C}$ à $-40^{\circ} \mathrm{C}$ et aux différentes températures de chauffage au cours de la cryodessiccation, à savoir de $80^{\circ} \mathrm{C}$ à $120^{\circ} \mathrm{C}$ afin d'évaluer la variation physico-chimique du milieu de culture, la survie et le pourcentage respectif des deux ferments lactiques présents pendant les traitements thermiques ainsi que leur viabilité au cours du vieillissement. La culture mixte se présente sous forme cryodesséché pour le stockage de longue durée.

\section{IDENTIFICATION DES MICRO-ORGANISMES}

\section{REACTIFS UTILISES}

Il s'agit des deux ferments lactiques : Streptococcus thermophilus - Lactobacillus helveticus, provenant de l'Ecole Polytechnique 
Fédérale de Zurich, régénérés et conservés dans notre laboratoire, sous forme de cultures pures et mixtes, à l'état frais et cryodesséchées.

\section{Streptococcus thermophilus}

\section{1) Origine}

C'est une souche pure de provenance de Zurich (Polytechnicum), sous l'immatriculation : Streptococcus thermophilus (M.T.I.) 4 - IV 1966.

\section{2) Aspect microscopique}

Les ferments lactiques Streptococcus thermophilus sont de forme sphérique en chaînettes régulières, d'une dimension de 2 microns environ, présentés toujours deux par deux.

Les streptocoques ont une coloration Gram positif.

\section{3) Caractéristiques taxonomiques}

Streptocoque thermophile : c'est un streptocoque homofermentatif très répandu dans les produits laitiers, exigeant de la nourriture azotée et vitaminique groupe $B$ et des acides aminés. La fermentation du Lactose est un caractère majeur des ferments lactiques Rogosa [10].

Caractères culturaux : Sur le milieu spécifique Man-Rogosa et Sharpe (M.R.S.) gélifié, les streptocoques donnent des petites colonies ponctifères. Les colonies se forment plus aisément en profondeur qu'en surface [1].

Voici la galerie d'identification des caractères biochimiques du Streptococcus thermophilus :

Culture à $10^{\circ} \mathrm{C}$ : négatif.

Culture à $37^{\circ} \mathrm{C}$ : positif.

Culture à $45^{\circ} \mathrm{C}$ : positif.

Survie à $63^{\circ} \mathrm{C}$ pendant $30 \mathrm{mn}$ : positif.

Fermentation du glucose et production de $\mathrm{CO}_{2}$ : négatif.

Réduction rapide du lait tournesolé (réduction avant coagula. tion) : négatif.

Production d'acétone : négatif.

Production d'ammoniac en hydrolysant de l'arginine : négatif.

Croissance en présence de chlorure de sodium :

$$
\begin{array}{r}
2 \text { p. } 100: \text { négatif } \\
4 \text { p. } 100: \text { négatif } \\
6,5 \text { p. } 100: \text { négatif }
\end{array}
$$


Sucres fermentés :

$$
\left.\begin{array}{l}
\begin{array}{l}
\text { lactose } \\
\text { glucose } \\
\text { galactose } \\
\text { saccharose }
\end{array} \\
\begin{array}{l}
\text { pentose } \\
\text { maltose } \\
\text { mannitol } \\
\text { salcine }
\end{array}
\end{array}\right\} \text { nositif }
$$

Résistance à la pénicilline : négatif.

Caractère pathogène : négatif.

\section{Lactobacillus helveticus}

1) Origine

C'est un ferment lactique thermophile, isolé du lait et du fromage. Largement répandu dans les produits laitiers [2].

De provenance de Zurich (Ecole Polytechnique) sous l'immatriculation : Lactobacillus helveticus 4-IX-1967.

\section{2) Aspect microscopique}

Les ferments lactiques Lactobacillus helveticus se présentent sous forme de bâtonnets de dimensions 0,7 à 0,9 microns de large et de 2 à 6 microns de long, seuls ou en chaînettes, non mobiles.

Les lactobacilles helvétiques ont une coloration Gram positif.

\section{3) Caractéristiques taxonomiques}

Lactobacille helvétique : c'est un lactobacille thermophile et homofermentatif. Il appartient au groupe sérologique A (Sharpe). Très répandu dans la présure naturelle et dans les fromages à pâte cuite. Il est microaérophile. Les températures optimales pour sa croissance sont $40^{\circ} / 42^{\circ} \mathrm{C}$, les températures minimales $20^{\circ} / 22^{\circ} \mathrm{C}$ et la température maximale $50^{\circ} \mathrm{C}$ [7].

Caractères culturaux :

- Milieu gélatiné : il ne croît pas sur ce milieu dans les températures nécessaires pour la gélatine.

- Milieu lactosé : il croît sur ce milieu et donne de petites colonies grises visqueuses.

- Le lait : il coagule le lait en produisant de l'acide lactique.

Voici la galerie d'identification des caractères biochimiques du Lactobacillus helveticus :

Culture à $15^{\circ} \mathrm{C}$ : négatif.

Culture à $45^{\circ} \mathrm{C}$ : positif. 
Résistance à $60^{\circ} \mathrm{C}$ pendant $90 \mathrm{mn}$ : positif.

Résistance à $65^{\circ} \mathrm{C}$ pendant $30 \mathrm{mn}$ : négatif.

Acide p. 100 dans le lait : 2,7.

Configuration de l'acide lactique : DL.

Production du $\mathrm{CO}_{2}$ à partir du glucose : négatif.

Production de $\mathrm{NH}_{3}$ à partir de l'arginine : négatif.

Croissance en présence de chlorure de sodium :

$$
\left.\begin{array}{l}
2 \text { p. } 100 \\
4 \text { p. } 100
\end{array}\right\} \text { négatif }
$$

Exigences nutritionnelles : Riboflavine et Pyridoxal.

Fermentation des sucres en produisant des acides : glucose, fructose, galactose, mannose, maltose et lactose (dextrine très peu).

L'acide lactique produit est inactif sur le plan optique.

Transformation des nitrates en nitrites : négatif.

Hydrolyse de l'esculine : négatif.

\section{TRAVAIL REALISE}

1) Préparation de lait à 14 p. 100 de matière sèche à base de lait écrémé en poudre. La quantité de lait est de 1,8 1, chauffé à l'autoclave $90^{\circ}$ à $100^{\circ} \mathrm{C}$ pendant $45 \mathrm{mn}$ [11].

Après l'autoclavage, on refroidit à $45^{\circ} \mathrm{C}$ sous l'eau courante, le ballon contenant $1,8 \mathrm{~kg}$ de lait ayant subi le traitement thermique et refroidi à $45^{\circ} \mathrm{C}$ pour être ensemencé.

2) L'ensemencement, se fait avec l'inoculum à partir des souches : streptocoque thermophile et lactobacille helvétique âgées de 18 à $24 \mathrm{~h}$. L'inoculum lui-même est conservé au froid à $4^{\circ} \mathrm{C}$, réchauffé à la température ambiante juste avant l'ensemencement. La dose d'ensemencement est de 5 p. 100.

La durée de l'étuvage est de 1 h 30 , à la température de $42^{\circ} \mathrm{C}$ [3]. Laisser refroidir le yaourt progressivement jusqu'à la phase stationnaire.

Les souches elles-mêmes sont conservées à l'état frais et cryodesséchées sous forme de culture pure et mixte.

3) Prélèvement, contrôles physico-chimiques et test de viabilité s'effectue systématiquement, pour mesurer le $\mathrm{pH}$ et l'acidité (acide lactique au g/l : degré Dornic) et test de coagulase, ce qui nous permet de déterminer la fin de la phase logarithmique de croissance des ferments lactiques ainsi que l'évolution du $\mathrm{pH}$ et la quantité de l'acide lactique dans le yaourt [4]. 
4) Congélation de cultures mixtes. Cette phase de l'opération est très importante, car nous cherchons à trouver les souches résistantes aux températures basses, à savoir : $-20^{\circ} \mathrm{C},-25^{\circ} \mathrm{C},-30^{\circ} \mathrm{C}$, $-35^{\circ} \mathrm{C}$ et $-40 \mathrm{C}$.

La vitesse de congélation varie suivant les températures de congélation appliquées, soit entre $8 \mathrm{~mm}$ et $3 \mathrm{~mm} / \mathrm{h}$ [6].

5) Prélèvement, contrôle physico-chimique et test de viabilité pour déterminer surtout le taux et le pourcentage respectifs des deux ferments lactiques présents (tests de revivification des microorganismes viables).

6) Cryodessiccation proprement dite. Les différentes phases de la cryodessiccation du yaourt (5) sont les suivantes :

$\left.\begin{array}{l}\text { - dessiccation primaire } \\ \text { - } \text { dessiccation secondaire }\end{array}\right\}$ sublimation

- dessiccation tertiaire

- séchage de finition $\{$ désorption

La durée totale de la cryodessiccation varie entre 8 et 9 h soit:

- sublimation $8 \mathrm{~h}$

) dessiccation primaire 5 à $6 \mathrm{~h}$

\{ dessiccation secondaire 2 à $3 \mathrm{~h}$

- désorption $1 / 2$ à $1 \mathrm{~h}$

$\left\{\begin{array}{l}\text { dessiccation tertiaire } \\ \text { ou }\end{array}\right.$

séchage de finition

Voici quelques exemples de durée de la cryodessiccation du yaourt $1800 \mathrm{~g}$ à base de ferments lactiques :

- streptocoque thermophile, lactobacille helvétique.

TABLEAU 1

\begin{tabular}{|c|c|c|c|c|c|}
\hline \multirow{2}{*}{$\begin{array}{l}\text { Température } \\
\text { de chauffage }\end{array}$} & \multicolumn{2}{|c|}{ Sublimation } & \multirow{2}{*}{$\begin{array}{c}\text { Désorption } \\
\text { Dessiccat. } \\
\text { tertiaire }\end{array}$} & \multirow{2}{*}{$\begin{array}{c}\text { Durée } \\
\text { du } \\
\text { cycle }\end{array}$} & \multirow{2}{*}{ Observations } \\
\hline & $\begin{array}{l}\text { Dessiccat. } \\
\text { primaire }\end{array}$ & $\begin{array}{l}\text { Dessiccat. } \\
\text { secondaire }\end{array}$ & & & \\
\hline $80^{\circ} \mathrm{C}$ & 5 & $21 / 2$ & $1 / 2 \mathrm{~h}$ & 8 & \\
\hline $90^{\circ} \mathrm{C}$ & 6 & 2 & $1 \mathrm{~h}$ & 9 & \\
\hline $100^{\circ} \mathrm{C}$ & $51 / 2$ & $21 / 4$ & $3 / 4 \mathrm{~h}$ & $8 \mathrm{~h} 30$ & \\
\hline $110^{\circ} \mathrm{C}$ & 6 & $13 / 4$ & $3 / 4 \mathrm{~h}$ & 8 h 30 & \\
\hline $120^{\circ} \mathrm{C}$ & $41 / 2$ & 3 & $1 / 2 \mathrm{~h}$ & 8 & \\
\hline
\end{tabular}

Cet exemple a été pris dans la série des yaourts congelés à la température de $-40^{\circ} \mathrm{C}$. 
Les chiffres ont été arrondis, mais la proportion a été respectée, chaque chiffre représente la moyenne de cinq essais.

7) Prélèvement, contrôle systématique par rapport aux produits congelés et frais ont été effectués.

La variation du $\mathrm{pH}$ est de l'ordre du dixième pour le même yaourt avant et après la cryodessiccation.

Voici quelques chiffres à titre d'exemple :

TABLEAU 2

\begin{tabular}{|c|c|c|c|c|c|c|}
\hline $\begin{array}{l}\text { Température } \\
\text { de chauffage }\end{array}$ & $80^{\circ} \mathrm{C}$ & $90^{\circ} \mathrm{C}$ & $100^{\circ} \mathrm{C}$ & $110^{\circ} \mathrm{C}$ & $120^{\circ} \mathrm{C}$ & Observations \\
\hline $\begin{array}{l}\text { Le } p H \underset{\text { drais }}{\text { du yaourt }} \\
\end{array}$ & 4,80 & 4,90 & 4,60 & 4,65 & 4,75 & \\
\hline $\begin{array}{l}\text { Le } \mathrm{pH}_{\text {cryodesséché }} \\
\text { cryaourt }\end{array}$ & 4,75 & 4,80 & 4,55 & 4,60 & 4,60 & \\
\hline
\end{tabular}

La température de congélation pour ces yaourts était de $-40^{\circ} \mathrm{C}$.

TABLEAU 3

\begin{tabular}{c|c|c|c|c|c|c}
\hline $\begin{array}{c}\text { Température } \\
\text { de chauffage }\end{array}$ & $80^{\circ} \mathrm{C}$ & $90^{\circ} \mathrm{C}$ & $100^{\circ} \mathrm{C}$ & $110^{\circ} \mathrm{C}$ & $120^{\circ} \mathrm{C}$ & Observations \\
\hline Le pH du yaourt & 4,8 & 4,75 & 4,65 & 4,7 & 4,7 & \\
\hline $\begin{array}{c}\text { Le pH du yaourt } \\
\text { cryodesséché } \\
\text { rehydraté }\end{array}$ & 4,7 & 4,7 & 4,55 & 4,55 & 4,6 & \\
\hline
\end{tabular}

La température de congélation pour ces yaourts était de $-35^{\circ} \mathrm{C}$.

TABLEAU 4

\begin{tabular}{|c|c|c|c|c|c|c|}
\hline $\begin{array}{l}\text { Température } \\
\text { de chauffage }\end{array}$ & $80^{\circ} \mathrm{C}$ & $90^{\circ} \mathrm{C}$ & $100^{\circ} \mathrm{C}$ & $110^{\circ} \mathrm{C}$ & $120^{\circ} \mathrm{C}$ & Observations \\
\hline $\begin{array}{l}\text { Le } \mathrm{pH} \mathrm{du} \text { yaourt } \\
\text { frais }\end{array}$ & 4,5 & 4,5 & 4,6 & 4,7 & 4,4 & \\
\hline $\begin{array}{l}\text { Le pH du yaourt } \\
\text { cryodesséché } \\
\text { rehydraté }\end{array}$ & 4,45 & 4,4 & 4,5 & 4,6 & 4,3 & \\
\hline
\end{tabular}

La température de congélation pour ces yaourts était de $-30^{\circ} \mathrm{C}$. 
TABLEAU 5

\begin{tabular}{|c|c|c|c|c|c|c|}
\hline $\begin{array}{l}\text { Température } \\
\text { de chauffage }\end{array}$ & $80^{\circ} \mathrm{C}$ & $90^{\circ} \mathrm{C}$ & $100^{\circ} \mathrm{C}$ & $110^{\circ} \mathrm{C}$ & $120^{\circ} \mathrm{C}$ & Observations \\
\hline $\begin{array}{c}\text { Le } \mathrm{pH} \text { du yaourt } \\
\text { frais }\end{array}$ & 4,7 & 4,8 & 4,65 & 4,8 & 4,8 & \\
\hline $\begin{array}{l}\text { Le } \mathrm{pH} \text { du yaourt } \\
\text { cryodesséché } \\
\text { rehydraté }\end{array}$ & 4,7 & 4,7 & 4,6 & 4,7 & 4,7 & \\
\hline
\end{tabular}

La température de congélation pour ces yaourts était de $-25^{\circ} \mathrm{C}$.

TABLEAU 6

\begin{tabular}{c|c|c|c|c|c|c}
\hline $\begin{array}{c}\text { Température } \\
\text { de chauffage }\end{array}$ & $80^{\circ} \mathrm{C}$ & $90^{\circ} \mathrm{C}$ & $100^{\circ} \mathrm{C}$ & $110^{\circ} \mathrm{C}$ & $120^{\circ} \mathrm{C}$ & Observations \\
\cline { 2 - 4 } $\begin{array}{c}\text { Le pH du yaourt } \\
\text { frais }\end{array}$ & 4,6 & 4,8 & 4,8 & 4,6 & 4,75 & \\
$\begin{array}{c}\text { Le pH du yaourt } \\
\text { cryodesséché } \\
\text { rehydraté }\end{array}$ & 4,55 & 4,8 & 4,6 & 4,45 & 4,7 & \\
\hline
\end{tabular}

La température de congélation pour ces yaourts était de $-20^{\circ} \mathrm{C}$.

Ce test de viabilité des ferments lactiques s'effectue d'une façon systématique sur les yaourts frais congelés et cryodesséchés.

Il en ressort que le taux des ferments lactiques ainsi que leur pourcentage respectif présents varient d'une façon sensible suivant la température de congélation et les conditions de la cryodessiccation. Le temps nécessaire pour la rehydratation et la reconstitution des yaourts cryodesséchés varie de 2 à $3 \mathrm{~h}$ selon le nombre des ferments lactiques revivifiables et leur pourcentage respectif.

\section{Analyses organoleptiques}

D'après les analyses organoleptiques qui se font d'une façon systématique, le yaourt a un goût agréable, parfois même méconnaissable par rapport à celui du frais.

Par contre la texture du yaourt rehydraté n'a pas la même fermeté que celle du frais. On peut faire une analogie entre le yaourt cryodesséché rehydraté et le yaourt dit goût bulgare (dont le $\mathrm{pH}$ est de l'ordre de 5 et dont la caséine a le point isoélectrique très élevé). 
Yaourt à base de ferments : Str. thermophilus et Lacto. helveticus, à 12 p. 100 de matière sèche

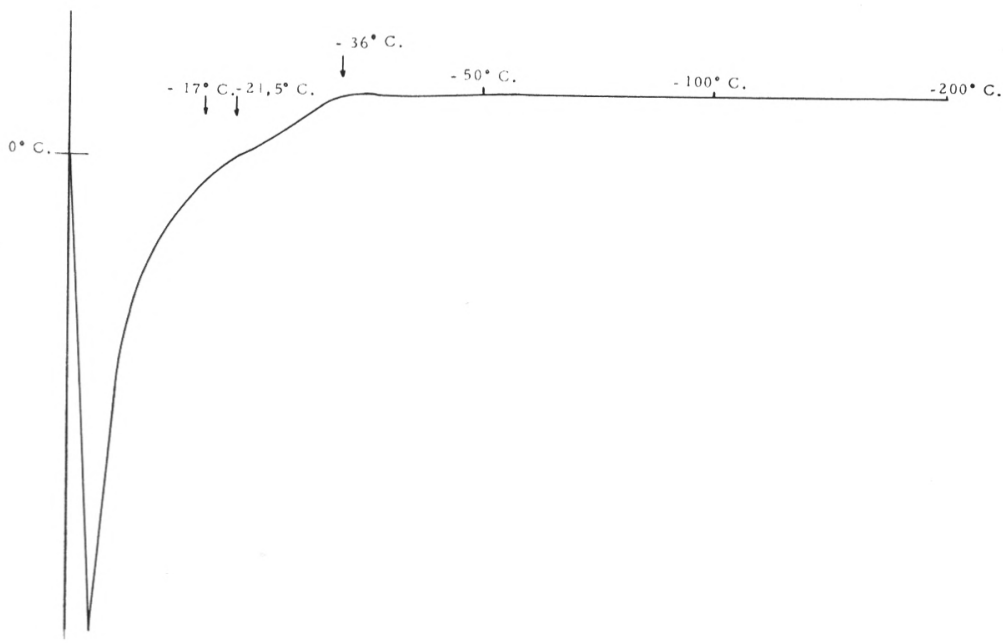

fig. 1

- Température de dévitrification - recristallisation est de $=-36^{\circ} \mathrm{C}$.

- Température de fusion commençante est de $=-21,5^{\circ} \mathrm{C}$.

- Température limite de travail est de $=-17^{\circ} \mathrm{C}$. 


\section{TABLEAU 7}

Influence de la température de congélation à $-20^{\circ} \mathrm{C}$ et de la cryo-dessiccation qui s'ensuit sur le taux de survie des deux ferments lactiques: Streptococcus thermophilus et Lactobacillus helveticus

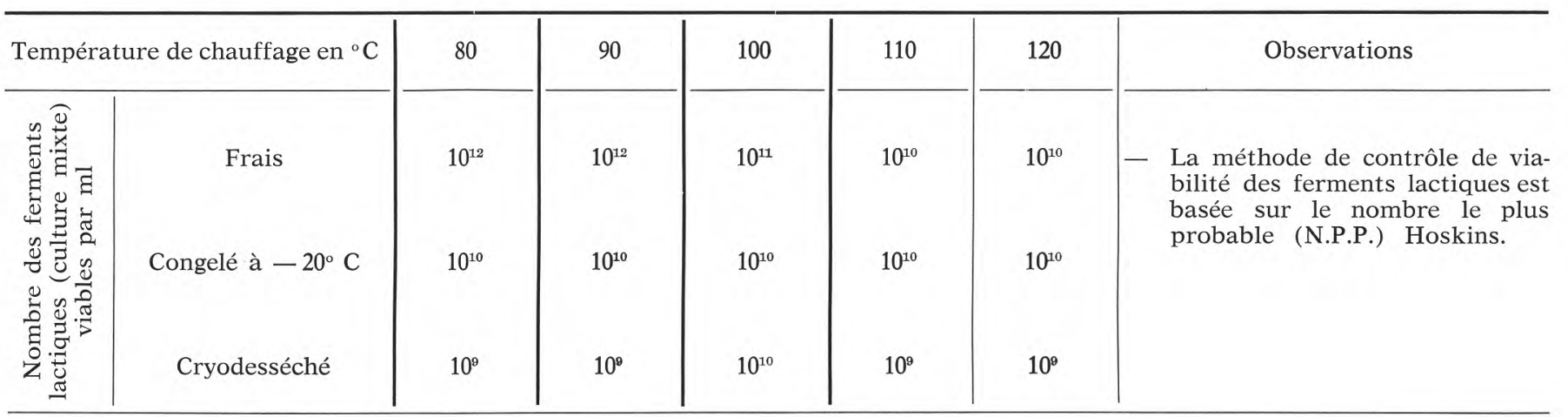

Le nombre des ferments lactiques viables dans le yaourt frais est de $10^{11}$ par $\mathrm{ml}$.

Le nombre des ferments lactiques viables dans le yaourt congelé à $-20^{\circ} \mathrm{C}$ est de $10^{10}$ par $\mathrm{ml}$.

Le nombre des ferments lactiques viables dans le yaourt cryodesséché est de $10^{9}$ par $\mathrm{ml}$. 
TABLEAU 8

Influence de la température de congélation à $-20^{\circ} \mathrm{C}$ et de la cryodessiccation qui s'ensuit sur le pourcentage des deux ferments lactiques (culture mixte) streptococcus thermophilus et Lactobacillus helveticus

\begin{tabular}{|c|c|c|c|c|c|c|c|c|}
\hline \multicolumn{3}{|c|}{ Température de chauffage en ${ }^{\circ} \mathrm{C}$} & 80 & 90 & 100 & 110 & 120 & Observations \\
\hline 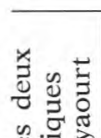 & Frais & $\begin{array}{c}\text { Strepto. } \\
\text { Lacto. }\end{array}$ & $\begin{array}{l}58 \\
42\end{array}$ & $\begin{array}{l}61 \\
39\end{array}$ & $\begin{array}{l}60 \\
40\end{array}$ & $\begin{array}{l}56 \\
44\end{array}$ & $\begin{array}{l}55 \\
45\end{array}$ & $\begin{array}{l}\text { - La méthode employée est l'exa- } \\
\text { men microscopique sur les frot- } \\
\text { tis colorés au bleu de méthy- } \\
\text { lène. }\end{array}$ \\
\hline 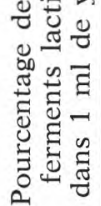 & $\begin{array}{c}\text { Congelé } \\
\text { à } \\
-20^{\circ} \mathrm{C} \\
\text { Cryo- } \\
\text { desséché }\end{array}$ & $\begin{array}{l}\text { Strepto. } \\
\text { Lacto. }\end{array}$ & $\begin{array}{l}55 \\
45\end{array}$ & $\begin{array}{l}62 \\
38\end{array}$ & $\begin{array}{l}57 \\
43\end{array}$ & $\begin{array}{l}55 \\
45\end{array}$ & $\begin{array}{l}53 \\
47\end{array}$ & $\begin{array}{l}\text { - Un contrôle parallèle par la mé- } \\
\text { thode de numération globale } \\
\text { avec le mélangeur de Potain et } \\
\text { la cellule de Malassez et Vignal, } \\
\text { a été effectué. }\end{array}$ \\
\hline
\end{tabular}

- Le pourcentage moyen des deux ferments lactiques respectifs est de 58 p. 100 pour le streptocoque et 42 p. 100 pour le lactobacille, dans la culture mixte fraîche.

- Le pourcentage moyen de ces deux ferments lactiques respectifs (culture mixte) congelés à $-20^{\circ} \mathrm{C}$ est de 56,4 p. 100 pour le streptocoque et 43,6 p. 100 pour le lactobacille.

- Le pourcentage moyen de ceux de ces deux ferments lactiques cryodesséchés rehydratés est de 55,4 p. 100 pour le streptocoque et 44,6 p. 100 pour le lactobacille. 
Influence de la température de congélation à $-25^{\circ} \mathrm{C}$ et de la cryodessiccation qui s'ensuit sur le taux de survie des deux ferments lactiques : Streptococcus thermophilus et Lactobacillus helveticus

\begin{tabular}{|c|c|c|c|c|c|c|c|}
\hline \multicolumn{2}{|c|}{ Température de chauffage en ${ }^{\circ} \mathrm{C}$} & 80 & 90 & 100 & 110 & 120 & Observations \\
\hline 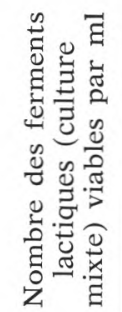 & Cryodesséché & $10^{10}$ & $10^{9}$ & $10^{11}$ & $10^{11}$ & $\begin{array}{l}10^{11} \\
10^{10} \\
10^{10}\end{array}$ & $\begin{array}{l}\text { - La méthode de contrôle de via- } \\
\text { bilité des ferments lactiques est } \\
\text { basée sur le nombre le plus } \\
\text { probable (N.P.P.) Hoskins. }\end{array}$ \\
\hline
\end{tabular}

Le nombre des ferments lactiques viables dans le yaourt frais est de $10^{11}$ par $\mathrm{ml}$.

Le nombre des ferments lactiques viables dans le yaourt congelé à $-25^{\circ} \mathrm{C}$ est de $10^{10} \mathrm{par} \mathrm{ml}$.

Le nombre des ferments lactiques viables dans le yaourt cryodesséché est de $10^{10}$ par ml. 


\section{TABLEAU 10}

Influence de la température de congélation à $-25^{\circ} \mathrm{C}$ et de la cryodessiccation qui s'ensuit sur le pourcentage des deux ferments lactiques (culture mixte) : Streptococcus thermophilus et Lactobacillus helveticus

\begin{tabular}{|c|c|c|c|c|c|c|c|c|}
\hline \multicolumn{3}{|c|}{ Température de chauffage en ${ }^{\circ} \mathrm{C}$} & 80 & 90 & 100 & 110 & 120 & Observations \\
\hline 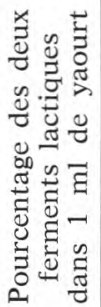 & $\begin{array}{c}\text { Congelé } \\
\text { à } \\
-25^{\circ} \mathrm{C} \\
\text { Cryo- } \\
\text { desséché }\end{array}$ & $\begin{array}{c}\text { strepto. } \\
\text { lacto. }\end{array}$ & $\begin{array}{l}54 \\
46\end{array}$ & $\begin{array}{l}58 \\
42\end{array}$ & $\begin{array}{l}51 \\
49\end{array}$ & $\begin{array}{l}53 \\
47\end{array}$ & $\begin{array}{l}57 \\
43\end{array}$ & $\begin{array}{l}\text { - La méthode employée est l'exa- } \\
\text { men microscopique sur les frot- } \\
\text { tis colorés au bleu de méthy- } \\
\text { lène. } \\
\text { - Un contrôle parallèle par la } \\
\text { méthode de numération globale } \\
\text { avec le mélangeur de Potain et } \\
\text { la cellule de Malassez et Vignal } \\
\text { a été effectué. }\end{array}$ \\
\hline
\end{tabular}

- Le pourcentage moyen des deux ferments lactiques respectifs est de 54,4 p. 100 pour le streptocoque et 45,2 p. 100 pour le lactobacille, dans la culture mixte fraîche.

- Le pourcentage moyen de ces deux ferments lactiques respectifs (culture mixte) congelés à - $25^{\circ} \mathrm{C}$ est de 54,6 p. 100 pour le streptocoque et 45,4 p. 100 pour le lactobacille.

- Le pourcentage moyen de ces deux ferments lactiques respectifs cryodesséchés rehydraté est de 49,5 p. 100 pour le streptocoque et 50,6 p. 100 pour le lactobacille. 


\section{TABLEAU 11}

Influence de la température de congélation à $-30^{\circ} \mathrm{C}$ et de la cryodessiccation qui s'ensuit sur le taux de survie des deux ferments lactiques: Streptococcus thermophilus et Lactobacillus helveticus

\begin{tabular}{|c|c|c|c|c|c|c|c|}
\hline \multicolumn{2}{|c|}{ Température de chauffage en ${ }^{\circ} \mathrm{C}$} & 80 & 90 & 100 & 110 & 120 & Observations \\
\hline 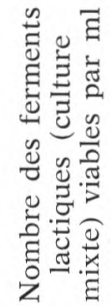 & $\begin{array}{c}\text { Congelé à }-30^{\circ} \mathrm{C} \\
\text { Cryodesséché }\end{array}$ & $10^{10}$ & $10^{10}$ & $10^{10}$ & $\begin{array}{l}10^{10} \\
10^{10}\end{array}$ & $\begin{array}{l}10^{10} \\
10^{10}\end{array}$ & $\begin{array}{l}\text { - La méthode de contrôle de via- } \\
\text { bilité des ferments lactiques est } \\
\text { basée sur le nombre le plus } \\
\text { probable (N.P.P.) Hoskins. }\end{array}$ \\
\hline
\end{tabular}

Le nombre des ferments lactiques viables dans le yaourt frais est de $10^{10}$ par ml.

Le nombre des ferments lactiques viables dans le yaourt congelé à $-30^{\circ} \mathrm{C}$ est de $10^{10}$ par $\mathrm{ml}$.

Le nombre des ferments lactiques viables dans le yaourt cryodesséché est de $10^{10}$ par $\mathrm{ml}$. 
TABLEAU 12

Influence de la température de congélation à $-30^{\circ} \mathrm{C}$ et de la cryodessiccation qui s'ensuit sur le pourcentage des deux ferments lactiques (culture mixte) : Streptococcus thermophilus et Lactobacillus helveticus

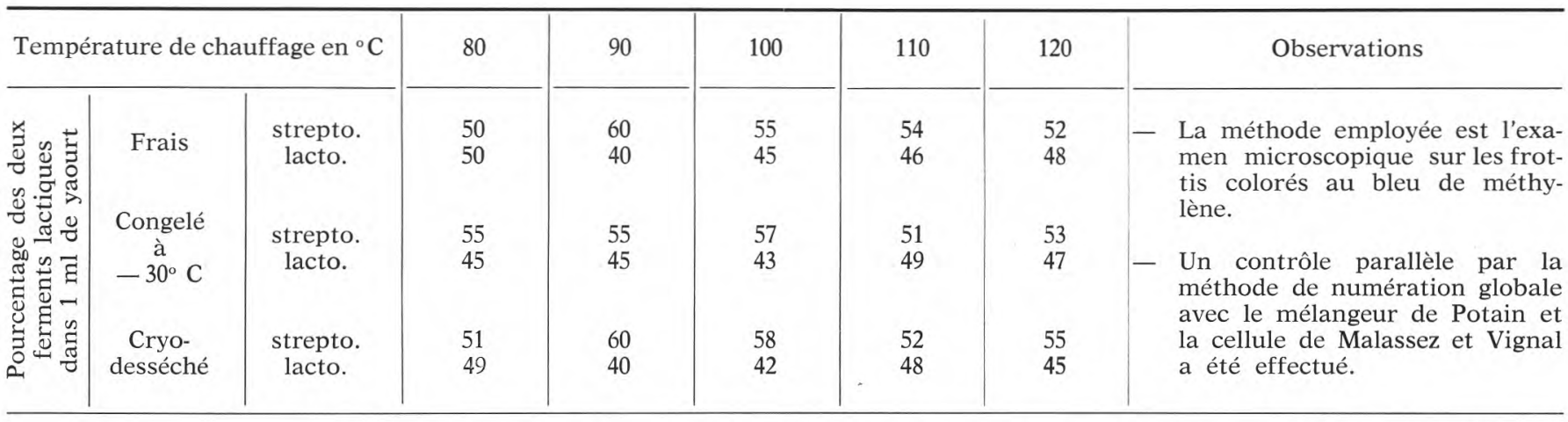

- Le pourcentage moyen des deux ferments lactiques respectifs est de 54,2 p. 100 pour le streptocoque et 45,8 p. 100 pour le lactobacille, dans la culture mixte fraîche.

- Le pourcentage moyen de ces deux ferments lactiques respectifs (culture mixte) congelés à - $30^{\circ} \mathrm{C}$ est de $54,2 \mathrm{p}$. 100 pour le streptocoque et 45,8 pour le lactobacille.

- Le pourcentage moyen de ces deux ferments lactiques respectifs cryodesséchés rehydratés est de 55,2 p. 100 pour le streptocoque et 44,8 p. 100 pour le lactobacille. 
Influence de la température de congélation à $-35^{\circ} \mathrm{C}$ et de cryodessiccation qui s'ensuit sur le taux de survie des deux ferments lactiques : Streptococcus thermophilus et Lactobacillus helveticus

\begin{tabular}{|c|c|c|c|c|c|c|c|}
\hline \multicolumn{2}{|c|}{ Température de chauffage en ${ }^{\circ} \mathrm{C}$} & 80 & 90 & 100 & 110 & 120 & Observations \\
\hline 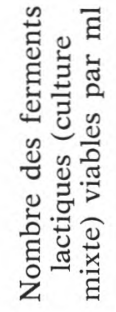 & Cryodesséché & $10^{10}$ & $10^{11}$ & $10^{10}$ & $10^{11}$ & $\begin{array}{l}10^{10} \\
10^{10}\end{array}$ & $\begin{array}{l}\text { - La méthode de contrôle de via- } \\
\text { bilité des ferments lactiques } \\
\text { (culture mixte) est basée sur le } \\
\text { nombre le plus probable (N.P.P.) } \\
\text { Horkins. }\end{array}$ \\
\hline
\end{tabular}

Le nombre des ferments lactiques viables dans la culture fraîche est de $10^{11}$ par ml.

Le nombre des ferments lactiques viables dans la culture mixte congelée à $-35^{\circ} \mathrm{C}$ est de $10^{10}$ par ml.

Le nombre des ferments lactiques viables dans la culture mixte cryodesséchée est de $10^{10} \mathrm{par} \mathrm{ml}$. 


\section{TABLEAU 14}

Influence de la température de congélation à $-35^{\circ} \mathrm{C}$ et de la cryodessiccation qui s'ensuit sur le pourcentage des deux ferments lactiques (culture mixte) : Streptococcus thermophilus et Lactobacillus helveticus

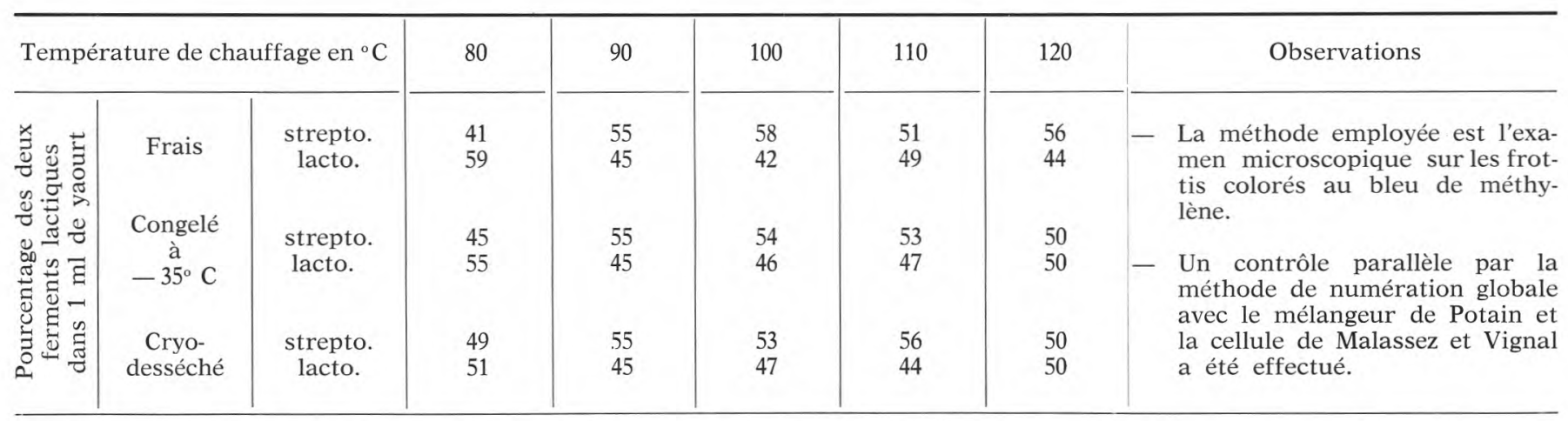

- Le pourcentage moyen des deux ferments lactiques respectifs est de 52,2 p. 100 pour le streptocoque et 47,8 p. 100 pour le lactobacille, dans la culture mixte fraîche.

- Le pourcentage moyen de ces deux ferments lactiques respectifs (culture mixte) congelés à $-35^{\circ} \mathrm{C}$ est de 51,4 p. 100 pour le streptocoque et 48,6 p. 100 pour le lactobacille.

- Le pourcentage moyen de ces deux ferments lactiques respectifs cryodesséchés rehydratés est de 52,6 p. 100 pour le streptocoque et 47,4 p. 100 pour le lactobacille. 


\section{TABLEAU 15}

Influence de la température de congélation à $-40^{\circ} \mathrm{C}$ et de la cryodessiccation qui s'ensuit sur le taux de survie des deux ferments lactiques : Streptococcus thermophilus et Lactobacillus helveticus

\begin{tabular}{|c|c|c|c|c|c|c|c|}
\hline \multicolumn{2}{|c|}{ Température de chauffage en ${ }^{\circ} \mathrm{C}$} & 80 & 90 & 100 & 110 & 120 & Observations \\
\hline 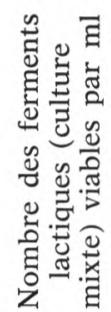 & $\begin{array}{c}\text { Congelé à }-40^{\circ} \mathrm{C} \\
\text { Cryodesséché }\end{array}$ & $\begin{array}{l}10^{10} \\
10^{10}\end{array}$ & $10^{11}$ & $10^{11}$ & $10^{11}$ & $\begin{array}{l}10^{11} \\
10^{10} \\
10^{10}\end{array}$ & $\begin{array}{l}\text { - La méthode de contrôle de via- } \\
\text { bilité des ferments lactiques est } \\
\text { basée sur le nombre le plus } \\
\text { probable (N.P.P.) Hoskins. }\end{array}$ \\
\hline
\end{tabular}

Le nombre des ferments lactiques viables dans le yaourt frais est de $10^{11}$ par ml.

Le nombre des ferments lactiques viables dans le yaourt congelé à $-40^{\circ} \mathrm{C}$ est de $10^{10}$ par $\mathrm{ml}$.

Le nombre des ferments lactiques viables dans le yaourt cryodesséché est de $10^{10}$ par ml. 
TABLEAU 16

Influence de la température de congélation à $-40^{\circ} \mathrm{C}$ et de la cryodessiccation qui s'ensuit sur le pourcentage des deux ferments lactiques (culture mixte) : Streptococcus thermophilus et Lactobacillus helveticus

\begin{tabular}{|c|c|c|c|c|c|c|c|c|}
\hline \multicolumn{3}{|c|}{ Température de chauffage en ${ }^{\circ} \mathrm{C}$} & 80 & 90 & 100 & 110 & 120 & Observations \\
\hline 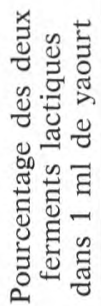 & $\begin{array}{c}\text { Congelé } \\
\text { à } \\
-40^{\circ} \mathrm{C} \\
\text { Cryo- } \\
\text { desséché }\end{array}$ & $\begin{array}{l}\text { strepto. } \\
\text { lacto. }\end{array}$ & $\begin{array}{l}56 \\
44\end{array}$ & $\begin{array}{l}43 \\
57\end{array}$ & $\begin{array}{l}50 \\
50\end{array}$ & $\begin{array}{l}52 \\
48\end{array}$ & $\begin{array}{l}47 \\
53\end{array}$ & $\begin{array}{l}\text { - La méthode employée est l'exa- } \\
\text { men microscopique sur les frot- } \\
\text { tis colorés au bleu de méthy- } \\
\text { lène. } \\
\text { - Un contrôle parallèle par la } \\
\text { méthode de numération globale } \\
\text { avec le mélangeur de Potain et } \\
\text { la cellule de Malassez et Vignal } \\
\text { a été effectué. }\end{array}$ \\
\hline
\end{tabular}

- Le pourcentage moyen des deux ferments lactiques respectifs est de 51,8 p. 100 pour le streptocoque et 48,2 p. 100 pour le lactobacille, dans la culture mixte fraîche.

- Le pourcentage moyen de ces deux ferments lactiques respectifs (culture mixte) congelés à $-40^{\circ} \mathrm{C}$ est de 50,6 p. 100 pour le streptocoque et 49,4 p. 100 pour le lactobacille.

- Le pourcentage moyen de ces deux ferments lactiques respectifs cryodesséchés rehydratés est de 52,2 p. 100 pour le streptocoque et 47,8 p. 100 pour le lactobacille. 


\section{Graphique $n^{\circ} 1$}

\section{- - - Congelé}

......... Cryodesséché

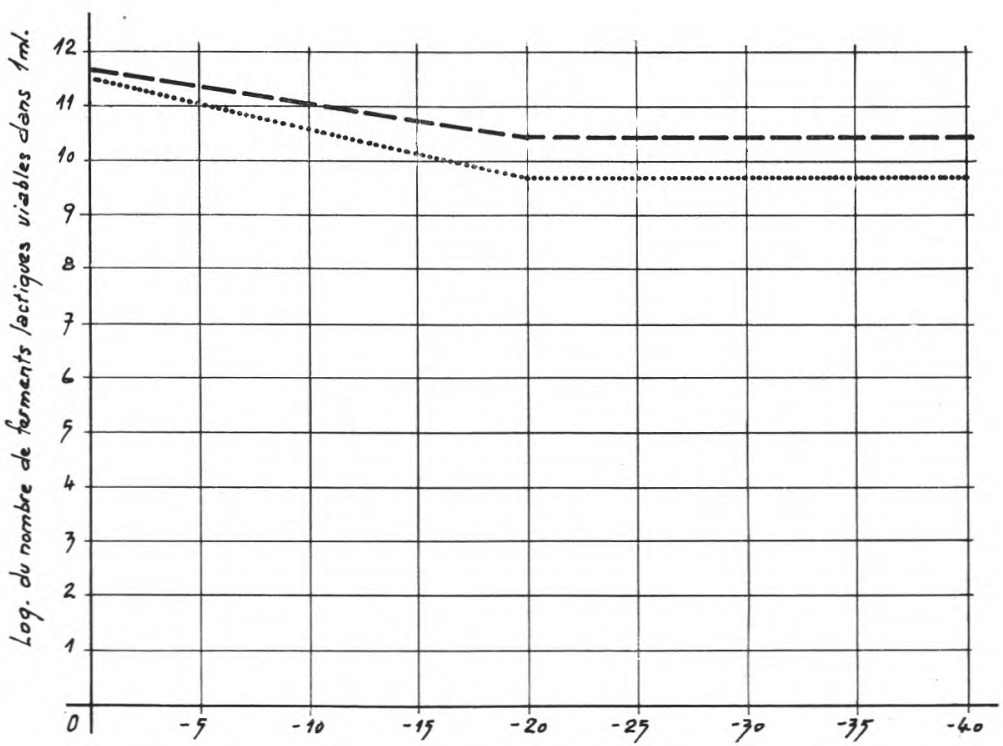

Température de congélation en degrés centigrades

- Influence des basses températures sur le taux de survie des ferments lactiques : Streptococcus thermophilus et Lactobacillus helveticus, dans le yaourt helvétique avant la phase stationnaire.

- On remarque le seuil de sensibilité des ferments lactiques aux températures basses dans le yoghourt avant la phase stationnaire. La perte des ferments lactiques se stabilise à partir de $-25^{\circ} \mathrm{C}$. Ce graphique a été établi sur la moyenne de 5 courbes pour chaque température de congélation, sauf pour le yoghourt helvétique frais dont le taux de population a été calculé sur une moyenne de 25 échantillons. 


\section{Graphique $n^{\circ} 2$}
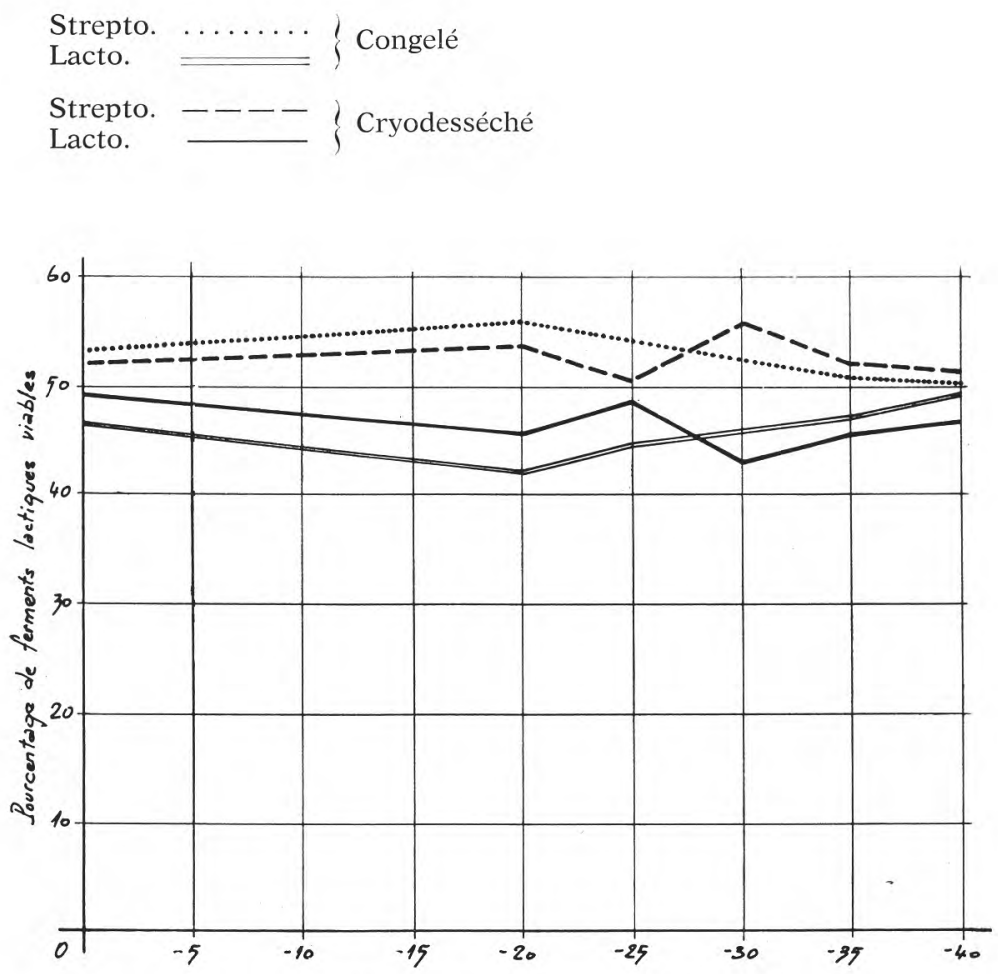

Température de congélation en degrés centigrades

- Influence des basses températures sur le pourcentage des ferments lactiques Streptococcus thermophilus et Lactobacillus helveticus dans le yoghourt helvétique avant la phase stationnaire.

- L'écart du pourcentage entre les ferments lactiques a tendance à s'affaiblir à partir de $-25^{\circ} \mathrm{C}$, ce graphique a été établi sur la moyenne de 5 courbes, sauf pour le yoghourt helvétique frais dont le pourcentage respectif des ferments lactiques présents a été calculé sur une moyenne de 25 échantillons. 


\section{CONCLUSIONS}

Dans cette partie de notre expérimentation, nous avons choisi les deux ferments lactiques :

- Streptococcus thermophilus,

- Lactobacillus helveticus,

pour étudier le comportement et la viabilité de cette culture mixte aux basses températures, à savoir : $-20^{\circ} \mathrm{C},-25^{\circ} \mathrm{C},-30^{\circ} \mathrm{C},-35^{\circ} \mathrm{C}$ et $-40^{\circ} \mathrm{C}$.

Le yaourt ordinaire est une symbiose des deux ferments lactiques suivantes : Streptococcus thermophilus et Lactobacillus bulgaricus, que nous avons étudiés précédemment.

En effet, nous avons obtenu des résultats reproductibles prouvant la sensibilité des ferments lactiques étudiés, en fonction des températures basses étudiées.

Il en ressort que le froid a un effet relativement important sur la viabilité de nos ferments lactiques. Cet effet nuisible est plus fort sur Lactobacillus helveticus, car celui-ci subit une perte plus importante que Streptococcus thermophilus. La disproportion entre les deux ferments lactiques présents varie entre 2 et 5 p. 100 , suivant la température de congélation.

Nous avons essayé de classer quantitativement en deux catégories les influences des températures basses :

$1^{\circ}$ La température de congélation à $-20^{\circ} \mathrm{C}$ a un rôle relativement considérable sur le taux des deux ferments lactiques viables.

$2^{\circ}$ Les températures basses de $-25^{\circ} \mathrm{C},-30^{\circ} \mathrm{C},-35^{\circ} \mathrm{C}$ et $-40^{\circ} \mathrm{C}$, ne modifient pas le taux et le pourcentage respectif des ferments lactiques présents.

En effet, l'écart du pourcentage respectif des ferments lactiques présents commence à partir de la température de congélation de $-20^{\circ} \mathrm{C}$. Cependant l'écart du pourcentage s'affaiblit à partir de $-25^{\circ} \mathrm{C}$ de sorte que celui-ci est négligeable à $-35^{\circ} \mathrm{C}$ et $-40^{\circ} \mathrm{C}$.

On peut en déduire que l'effet du froid sur le taux et le pourcentage respectif des ferments lactiques présents est nettement moins important par rapport aux autres ferments lactiques étudiés précédemment, à savoir :

- Lactobacillus bulgaricus,

- Lactobacillus acidophilus.

Cette résistance du Lactobacillus helveticus au froid est due à plusieurs raisons, entre autres :

- la résistance physiologique de ce ferment lactique,

- l'interaction entre les deux ferments lactiques. 
En effet, il doit exister un effet stimulant de la part de streptocoque par son métabolisme sur le lactobacillus helvétique.

Les produits du métabolisme du streptocoque ont été mis en évidence pour la première fois par Veringa [12 et 13]. C'est une substance chimique du genre de l'acide formique, celui-ci a un rôle de croissance et de protection pour le Lactobacillus helvétique.

$\mathrm{Au}$ cours de la cryodessiccation, l'écart du pourcentage entre les Streptococcus thermophilus et les Lactobacillus helveticus à $-25^{\circ} \mathrm{C}$ diminue de sorte qu'à $-35^{\circ} \mathrm{C}$ et à $-40^{\circ} \mathrm{C}$ l'écart est insignifiant.

De même, la symbiose entre ces deux ferments :

- Streptococcus thermophilus,

- Lactobacillus helveticus,

de sorte qu'ils résistent mieux aux températures basses par rapport aux autres cultures mixtes étudiées précédemment :

- Streptococcus thermophilus et Lactobacillus bulgaricus,

- Streptococcus lactis et Lactobacillus acidophilus,

car le pourcentage de leur taux est de 12 à 15 p. 100 plus fort que pour les autres ferments lactiques. D'après nos constatations, le taux de survie et la viabilité des ferments lactiques tels que : Streptococcus lactis, Lactobacillus bulgaricus et Lactobacillus acidophilus sont de l'ordre de 10 p. 100 dans les cultures mixtes cryodesséchées, tandis que le taux de population des Lactobacillus helveticus varie entre 10 à 15 p. 100 suivant les températures de congélation, ce taux étant de 12 p. 100 en moyenne.

Enfin, nous avons pu constater que la température optimale de la surface du yoghourt helvétique (culture mixte) cryodesséché pendant la dernière phase de cryodessiccation (dessiccation tertiaire) se situe entre $30^{\circ} \mathrm{C}$ et $35^{\circ} \mathrm{C}$, cette température de finition a un rôle efficace sur le taux de viabilité des ferments lactiques présents dans la culture mixte cryodesséchée.

\section{R és u m é}

D’après nos expérimentations sur le yoghourt helvétique, celui-ci étant à base des ferments lactiques suivants :

- Streptococcus thermophilus,

- Lactobacillus helveticus,

aux différentes températures de congélation et de la cryodessiccation qui s'ensuit, il en ressort que le lactobacille helvétique résiste relativement mieux aux basses températures que les lactobacilles bulgares et acidophiles, car le pourcentage de son taux de survie est de 
12 à 15 p. 100 , tandis que celui-ci est de 10 p. 100 pour les deux ferments lactiques ci-dessus énumérés.

Enfin, nous avons pu constater que la température optimale de la surface du yoghourt helvétique pendant la dernière phase de cryodessiccation se situe entre $-30^{\circ} \mathrm{C}$ et $35^{\circ} \mathrm{C}$. Celle-ci a un rôle efficace sur le taux de viabilité des ferments lactiques dans la culture mixte cryodesséchée.

\section{S u m m a ry}

During our experimentations on helvetic yoghurt : Str. thermophilus and Lacto. helveticus, under different freezing temperatures and freeze-drying, we found out that Lactobacillus helveticus had better resistance during freezing and freeze-drying process.

The percentage of viability for this Lacto-acid-bacteria is $12-15$ p. 100 and only 10 p. 100 for Lactobacillus bulgaricus and Lactobacillus acidophilus.

Finaly, we appreciated that the temperature for freeze-drying process in the end of cycle is situated between $+30^{\circ} \mathrm{C}$ and $+35^{\circ} \mathrm{C}$. This temperature has influence on viability of Lacto-acid-bacteria.

\section{Remerciements}

Nous remercions $M$. le $\mathrm{Pr}$ Thieulin pour les précieux conseils qu'il a bien voulu nous donner pour la rédaction de cet article, ainsi que $M$. le $\mathrm{Pr}$ Keilling, notre Maître et Conseiller permanent. Nous remercions également $M$. le Pr Ulrich pour son concours scientifique.

\section{Bibliographie}

[1] Alais (Ch.) (1965). - Science du lait. Editions Sep., Paris.

[2] Bergey (D. H.) (1957). - Manuel of determinative Bacteriology, 7 th, édition Baltimore the, Williams and Wilkins Compagny, 521-545.

[3] Gavin (M.) (1968). - Thèse Doctorat. La lyophilisation des cultures de yoghourt, présentée à l'Ecole Polytechnique Fédérale, Zurich (Suisse).

[4] Gehriger (1968). - These Doctorat. Die Herstellung Lyophilisierter Kultuturen von Lactobacillus helveticus und Streptococcus thermophilus und ihre verwendung in der Emmentoler Kaserei Eidgesnossischen Technschen Hoschschule. Zurich (Suisse).

[5] JABARIT (A.). - Influence de la congélation et de la cryo-dessiccation sur le taux de survie et le pourcentage des ferments lactiques dans le yaourt. SOCALTRA, Centre de Recherches, 128, bd Victor-Hugo (92) Clichy. Le Lait, 1969, 483-4, 160.

[6] Jabarit (A.) (1969). - Influence de la congélation et de la cryodessiccation sur le taux de survie et le pourcentage des ferments lactiques dans le 
bioghourt. SEPIAL, Centre de Recherches, 128, bd Victor-Hugo (92) Clichy. Le Lait, 1969, 488, 530.

[7] Orla-Jenson (S.) (1919). - Mem. Acad. Roy. Sci. Lettres, Copenhague. Sci 8, V 79-197.

[8] Pette (J. W.) et Lolkema (I. H.) (1951 a). - Neth. Milk and Dairy J., 5, 14-26.

[9] Pette (J. W.) et Lolkema (I. H.) (1951 b). - Neth. Milk and Dairy J., 5, 27-45.

[10] Rogosa (M.) (1943). - Journal of Bacteriology, 46, 436.

[11] Störgards (S.) et Andelsen (S.) (1964). - Traitement thermique du lait avant l'ensemencement. Rapport général sur les journées d'études de la F.I.L. à Paris, $\mathrm{n}^{\circ} \quad 6,65$.

[12] Veringa (H. A.), Galesloot (The E) et Davelaar (1968). - Symbiose dans le yoghourt (II), isolation et identification du facteur de croissance pour Lactobacillus bulgaricus produit par Streptococcus thermophilus. Neth. Milk and Dairy Journal, 22, 114.

[13] Veringa (H. A.), Galesloot (The E.) et Hassing (F.) (1968). — La symbiose dans le yoghourt, la stimulation du Lactobacillus bulgaricus par un facteur produit par le Streptococcus thermophilus. Neth. Milk and Dairy Journal. 